\title{
Implementation of Population Algorithms to Minimize Power Losses and Cable Cross-Section in Power Supply System
}

\author{
V. Z. Manusov, P. V. Matrenin, E. S. Tretiakova
}

First-Third Department of Industrial Power Supply Systems, Novosibirsk State Technical University, Prospekt K. Marksa, Novosibirsk, 630073, Russia

\begin{tabular}{l} 
Article Info \\
\hline Article history: \\
Received May 27, 2016 \\
Revised Nov 6, 2016 \\
Accepted Nov 30, 2016 \\
\hline
\end{tabular}

Keyword:

Active power losses

Cable cross-sections

Deep compensation

Population-based optimization

Power-supply systems

\begin{abstract}
The article dues to the arrangement of the reactive power sources in the power grid to reduce the active power losses in transmission lines and minimize cable cross-sections of the lines. The optimal arrangement is considered from two points of view. In the first case, it is possible to minimize the active power losses only. In the second case, it is possible to change the cross-sections of the supply lines to minimize both the active power losses and the volume of the cable lines. The sum of the financial cost of the active power losses, the capital investment to install the deep reactive power compensation, and cost of the cable volume is introduced as the single optimization criterion. To reduce the losses, the deep compensation of reactive power sources in nodes of the grid are proposed. This optimization problem was solved by the Genetic algorithm and the Particle Swarm optimization algorithm. It was found out that the deep compensation allows minimizing active power losses the cable cross-section. The costeffectiveness of the suggested method is shown. It was found out that optimal allocation of the reactive power sources allows increasing from $9 \%$ to $20 \%$ the financial expenses for the enterprise considered.
\end{abstract}

Copyright (c) 2016 Institute of Advanced Engineering and Science. All rights reserved.

\section{Corresponding Author:}

E. S. Tretiakova,

First-Third Department of Industrial Power Supply Systems,

Novosibirsk State Technical University,

Prospekt K. Marksa, Novosibirsk-630073, Russia.

Email: elena.tretyakova.1966@list.ru

\section{INTRODUCTION}

Deep reactive power compensation increases the efficiency of power transmission and decreases overall energy consumption. It is necessary because transmission of the reactive current from active power generators to power consumers is unprofitable.

Power lines with voltage up to $10 \mathrm{kV}$ are the most significant part of distributed networks of industrial enterprises. Due to extensive branching and length of power lines with voltage up to $10 \mathrm{kV}$, low-voltage networks are characterized by high losses of power. Reducing the consumption of reactive power allows industrial plants to degrade the existing capacity constraints in distribution power systems, as well as lower the cost of energy consumption. In this paper, the deep compensation of the reactive power is considered. The efficiency of the compensation depends on the allocation of reactive power compensation units at the nodes of a network.

The paper is organized as follows. Section II gives the brief overview of the deep compensation in power supply system under consideration and presents the mathematical model of the optimization problem. Section III describes the population-based optimization algorithms applied: the Genetic algorithm (GA) and the Particle Swarm Optimization (PSO). Section IV shows the implemented interface between the model of the optimization problem and the optimization algorithms and gives the experiments evidence. Section V concludes the paper. 


\section{PROBLEM DESCRIPTION}

\subsection{Statement of the problem}

Nowadays there is no universal method of finding the optimal allocation of the reactive power compensation units in nodes of the power-supply system. This optimization task is nonlinearity, no differentiability, has multiple extremes and high computational complexity. In addition, the models of such problems are characterized by a complex structure and topology of the search space. In power supply system for such optimization problems the Artificial Intelligence methods, such as Genetic algorithms [1-4], Swarm Intelligence [5-7], are used successfully.

Reactive power compensation into power supply systems is necessary because transmission of the reactive current from active power sources (generators) to power consumers is unprofitable. It is due to the fact that the reactive current occupies part of the cross section of a conductor of electric power lines and creates therein the same losses of active power $\left(I^{2} R\right)$ as the active current increasing simultaneously the current density into the conductor $\left(\mathrm{A} \cdot \mathrm{mm}^{2}\right)$.

In this paper, the calculations were made for a power supply grip of one section of internal consumption substation of an industrial enterprise, where the climatic conditions for Equationuipment are strictly regulated. Motors of pumps, fans and compressor are the main consumers, and a length of cable lines are several hundreds of meters. The geographical arrangement of grid's elements depends on the arrangement of the processing Equationuipment, so, it is impossible to reduce the length of the supply.

In this study, the power supply system of a uranium production plant in the town of Angarsk is considered. The power supply system represents 400 Voltages substation comprising 4 sections, and the arrangement of each section is radial.

The power-supply system of the each section has 10 power distribution points, 2 pumps Equationuipped with motors of $132 \mathrm{~kW}$ and 2 supply lines for the compressors. Every supply line consists of 13 compressors $(9 \mathrm{~kW})$ that are supplied with a ribbon cable. Active power losses in transmission lines of this network are high due to the network having a lot of branches and large distances between the nodes. It is suggested to install RPCUs close to the power distribution points, close to control cabinets of pumps and close to the control rack of the first compressor at the supply lines. The calculation of maximum load showed that now ratio of consumption of active and reactive powers of the system $(\operatorname{tg} \varphi)$ is 0.55 . This fact indicates the low energy efficiency of supply system under consideration.

\subsection{The mathematical model}

Application of the first way results in to unload the network by reactive power and consEquationuently to increasing active power losses in the network by reason of reactive power transmission in the cable lines. The second method leads us to formulate an optimization problem. To create a mathematical model of the optimization problem, it is necessary to define, an optimization criterion, controlled variables, and constraints. The main aim of the optimization is to minimize active power losses. The dependent variables are the values of the RPCU power in the nodes.

$$
\begin{aligned}
& Z_{1}\left(Q_{x}\right)=c_{Q} Q_{\Sigma}\left(Q_{x}\right)+c_{P} T \Delta P\left(Q_{x}\right) \rightarrow \min , \\
& 0 \leq Q_{x i} \leq Q_{\max i}, i=1, \ldots, n .
\end{aligned}
$$

The components in the Equation 1 are determined as follows:

a. $Q_{x}=\left\{Q_{x 1}, Q_{x 2}, \ldots, Q_{x n}\right\}$;

b. $Q_{x i}$ power of the RPCU in $i$-th junction;

c. $Q_{\max }$ is reactive power of load in $i$-th junction;

d. $n$ is a quantity of considered junctions available for installation of the RPCUs;

e. $c_{Q}$ is cost of the RPCUs (per volt-ampere);

f. $Q_{\Sigma}\left(Q_{x}\right)$ is sum $Q_{x i}, i=1, \ldots, n$;

g. $c_{P}$ is cost of electric losses;

h. $\triangle P\left(Q_{x}\right)$ is total losses of active power within a network using powers of RPCU defined by $Q_{x}$.

i. $T$ is a considered operation interval expressed in hours at maximum load.

In addition, the allocation of the RPCUs close to the distribution points allows reducing cross-sections of the supply cables. Obviously, the current density in the power lines is reduced by the deep compensation. The conductor cross-section is selected on the basis of relationship $F=I / j_{\text {econ }}\left(j_{\text {econ }}\right.$ is a certain economic current density. As the current decreases, the cross-section of the conductors can be decreased too. At the same time, currents in switching devices are reduced. The main consumers of the power supply systems are pumps, motors, fans and compressors, their arrangement depends on the location of the Equationuipment, therefore minimizing the length of the supply cable lines is not possible, while they 
amount to hundreds of meters. While cables have a fixed length, the volume of cable production can be changed since the possible cable cross-sections depend on current transferred.

Thus, the formulation of the optimality criteria can be written as follow:

$$
Z_{2}\left(Q_{x}\right)=c_{Q} Q_{\Sigma}\left(Q_{x}\right)+c_{P} T \Delta P\left(Q_{x}\right)+c_{V} V\left(Q_{x}\right) \rightarrow \min ,
$$

where $c_{V} V\left(Q_{x}\right)$ is the total cost of the cable production volume.

The value $Z_{2}\left(Q_{x}\right)$ are evaluated by the following scheme:

1. Calculate the currents, voltages in the grid, and active power losses $\Delta P\left(Q_{x}\right)$ using the initial cross-sections

2. Summarize powers of the RPCUs $\mathrm{Q}_{\Sigma}\left(\mathrm{Q}_{\mathrm{x}}\right)$

3. Determine minimal possible cross-section of the main cable lines of the grid and find the total volume $\mathrm{V}\left(\mathrm{Q}_{\mathrm{x}}\right)$.

4. Recalculate the value of $\Delta \mathrm{P}\left(\mathrm{Q}_{\mathrm{x}}\right)$ again using the new cross-sections, since active power losses change after changing of cross-sections.

5. Finally, determine the $\mathrm{Z}_{2}\left(\mathrm{Q}_{\mathrm{x}}\right)$ using Equation 3 .

6. The connection between the algorithms and the optimization problem

\section{EVOLUTIONARY AND SWARM OPTIMIZATION}

\subsection{Evolutionary and Swarm optimization}

The population-based algorithms using principles of nature demonstrate the highest performance among the other stochastic optimization methods. Evolutionary and swarm methods are classified as so-called population-based methods since they use systems of agents. The term agent can be defined as a point $X$ into the decision space of the optimization problem defined as $f(X) \rightarrow$ extr. We divide Evolutionary and Swarm methods since the optimization process may be evolutionary or swarming [8]. The evolutionary process is based on the creation the new populations at every new step taking into account the experience (a number of last solutions of the optimization problem and the solutions' qualities) obtained by the previous steps, so this process similar to the natural selection. The Swarm process is based on the movements of the agents into the decision space using a number of rules and the interactions between the agents. In contrast to evolutionary algorithms, the agents are not created and not destroyed and the swarm population has not any centralized control system. The comparison evolutionary and swarm methods are presented in Table 1.

Table 1. Evolutionary and Swarm Optimization

\begin{tabular}{lll}
\hline \multicolumn{1}{c}{ Feature } & \multicolumn{1}{c}{ Evolutionary } & \multicolumn{1}{c}{ Swarm } \\
\hline Inspiration & Natural selection & Collective behavior of bees, flocks, ants, fishes, etc. \\
Each step & Creating new populations at every new & Movements using a number of rules and an indirect \\
& step & exchange of data between the agents \\
Control & Centralized control system & Decentralized control system \\
Examples & Genetic algorithm & Particle swarm optimization \\
& Genetic programming & Artificial bee colony optimization \\
& Differential evolution & Ant colony optimization \\
& & Bat algorithm \\
\hline
\end{tabular}

The main advantage of the population algorithms is the ability to explore the decision space automatically regardless of its dimension and topology, it results at rather quickly finding good solutions. In this paper, the Genetic algorithm and the Particle Swarm Optimization algorithms are applied. The descriptions detailed of these algorithms are easy to find by the literature (GA [9],[10], Swarm Intelligence [11], PSO [12-13]), therefore, this paper gives the brief description without the mathematical models.

\subsection{The Genetic algorithm}

The GA started to be used for solving optimization problems in 1960-70 after researches of Ingo Rechenberg and John Holland [10]. GA is based on the principles of the natural selection: inheritance (the transition characteristics from parent to progeny), mutation (the sudden change characteristics), selection (the choice of more suitable units) and crossing over (the interchange of corresponding characteristics) [9-10]. It is the evolutionary algorithm. Solutions of the optimization problem are recorded as vectors of values and called chromosomes. However, we suggest using the term agent for uniformity. The term agent is commonly used in the field of the Swarm Intelligence, but it can also be applied in describing the evolutionary algorithms. 
The process of optimization simulates the natural selection. Every agent $X$ in the population is evaluated by the value of optimization criterion, calculated as $f(X)$. First, the random population is generated and for each $X$ value $f(X)$ is calculated. Then the algorithm selects the agents for the next iteration, taking into account the $f(X)$ of each agent. Selected agents are combined and changed randomly, so the new population is created and for each new agent $f(X)$ is evaluated. While the stop criterion is not met, the selection, combining, etc. are re-start.

\subsection{The Particle Swarm Optimization algorithm}

The Particle Swarm Optimization algorithm is a one of the most commonly used Swarm Intelligence algorithms. PSO based on a bird flocks behavior and it was developed by J. Kennedy and R. Eberhart [12]. Bird flock acts coordinated according to a number of simple rules. Every bird (particle) coordinates the own movements with the flocks. In the PSO algorithm, every particle is denoted by coordinates $X$ and by the value of the criterion $f(X)$. The vector $X$ is the position of a particle, and a vector $V$ is a velocity of a particle. Initial values of $X$ and $V$ are random. The vectors $X$ and $V$ of all particles are updated according to a number of rules using the best position of a particle, the best position of the whole swarm, the inertia weights of the particles and the stochastic deviations.

\subsection{PSO parameter selection}

The PSO algorithms has 3 number of behavioural parameters $\alpha_{1}, \alpha_{2}, \omega$ and $v_{\max }$, which allow to control speed, performance and other features of the process of the PSO operation [13-14] The necessity of tuning the values of the parameters for a task solved is a significant imperfection of the PSO for its high effective realization [13], [15]. Manual changing of the parameters and usage of several predefined sets of the parameters are the easiest and the most prevalent ways to the parameters selection. The first way rEquationuires a low of time and does not ensure the effective solution. The second way consists in choosing the best parameters among the several usable sets by experiments. This way also limits does not ensure the effective solution since it is limited by set sets of the parameter used. The research [13] provides an overview of studies that deal with tuning PSO parameters. It points out a primitiveness and low efficiency of manual tuning, but at the same time, it criticizes more complex methods for their range of application limitation and excessive algorithm complication. Therefore, in this research, a technic of the meta-optimization [13-14] was applied. The meta-optimization allows tuning the behavioural parameters automatically.

\section{EXPERIMENT AND ANALYSIS}

\subsection{Application of the population-based optimization}

The interaction of the algorithm and the models of the optimization problems (Equation 1-3) can be described by the simple scheme. For each algorithm iteration and each agent of population:

1. The algorithm gives the new agent's position $X$.

2. The model of a power grid gets the $X$ and mapping it to the vector $Q_{x}$ and the value of the criterion $Z_{1}\left(Q_{x}\right)$ or $Z_{2}\left(Q_{x}\right)$ is determined.

3. The algorithm gives the value the criterion as $f(X)$.

4. When the steps 1-3 are carried out for all agents, the positions of the agents are changed by PSO or the new population of the agents are generated by GA; and the process is repeated.

Thus, the method of calculation of $Z_{1}\left(Q_{x}\right)$ and $Z_{2}\left(Q_{x}\right)$ can be anyone, regardless of the optimization algorithm and the optimization algorithm is independent of it. This approach allows us to apply different optimization methods easily and quickly. There are not close relationships between the model of the optimization problem and the optimization algorithms. It is possible due to the flexibility and adaptability of population-based algorithms to the conditions of the optimization problems. Thus, the calculation of a power grid can be carried out means a specialized software as the analysis of relationships between key indicators of the grid and values of variables and finding the optimal mode of the grid can be performed by a separate artificial intelligence software library.

In the case considered, it is necessary to map the vector $X$ and the vector $Q_{x}$ to apply the interface described above. In order to make application and description of the algorithms, it is posited that the search space of the algorithms is limited between 0.0 and 1.0 for each axis. The vector $X$ is used not as the RPCUs power vector $Q_{x}$, but as the coefficients vector to take into account the maximum allowable power of RPCU in the nodes.

$$
Q_{x i}=X_{i} \cdot Q_{\max i}, 0 \leqslant X_{i} \leqslant 1, i=1, \ldots, n
$$


Thus, the optimization algorithm is independent of criteria calculation. Due it, changes in the power grid do not cause the necessity of changing anything in the optimization method implementation. To consider the limitations of $\operatorname{tg} \varphi$ the penalty values are used. If the value of $\operatorname{tg} \varphi$ does not fit the limitations, then the value of the optimization criterion $\left(Z_{1}\left(Q_{x}\right)\right.$ or $\left.Z_{2}\left(Q_{x}\right)\right)$ is added to an extra-large penalty value.

\subsection{Experiment description}

The optimization problems (Equation $1 \& 2$ and Equation $2 \& 3$ ) were solved by GA and PSO. We used GA with the one-point crossover of two parents, the number of agents was 100, the mutation probability was $15 \%$ and the crossover probability was $80 \%$.

The heuristics parameters of PSO were selected by meta-optimization approach [12]. The PSO parameters were followed:
a. $\alpha_{1}=1.49$,
b. $\alpha_{2}=1.57$,
c. $\omega=0.87$,
d. $\quad v_{\max }=0.9$.

The number of the PSO agents was 100 too. As far as the both algorithms are stochastic, they give a non-deterministic various solution of the optimization problems from run to run. Therefore, the algorithms were run 10 times and then the best solution was selected for each algorithm as the resulting solutions. The computation times of the both algorithms were about the same (10000 iterations).

\subsection{Experiment results}

Table 2 shows the results obtained for of the problem of Equation 1\&2 when the arrangement of the RPCUs was optimizing without changing of the cable cross-section.

Table 2. The Results from Equation 1 and 2 without Changing the Cable Cross-Sections (4 years)

\begin{tabular}{ccccc}
\hline Algorithm & Criterion $Z_{1}(€)$ & $\begin{array}{c}\text { Active power } \\
\text { losses }(\mathrm{kW})\end{array}$ & $\begin{array}{c}\text { Total power of } \\
\text { RPCUs }(\mathrm{kvar})\end{array}$ & $\operatorname{tg} \varphi$ \\
\hline without optimization & 43254 & 40.5 & 0 & 0.55 \\
GA & 38602 & 32.9 & 1093 & 0.15 \\
PSO & 37690 & 32.1 & 1075 & 0.1 \\
\hline
\end{tabular}

It was found out that the solution by PSO is better than ones by GA both in terms of the necessary total power of installed reactive power sources and in terms of the active power losses. However, if we consider the quantitative values of the optimality criterion, it is obvious that difference between the two algorithms considered is quite small, it is only $2.4 \%$. The analysis of the financial difference for the whole power supply system of the industrial enterprise considered is presented in Table 3 (the period is 4 years too).

Table 3. The Comparison of the Performance of the Algorithms

\begin{tabular}{cccc}
\hline Algorithm & $\begin{array}{c}\text { Decrease of the losses } \\
\Delta P(\%)\end{array}$ & $\begin{array}{c}\text { Active power losses for } \\
\text { the section }(€)\end{array}$ & $\begin{array}{c}\text { Active power losses cost } \\
\text { for the whole system }(€)\end{array}$ \\
\hline without optimization & 0 & 86508 & 346032 \\
GA & 18.8 & 70274 & 281098 \\
PSO & 20.7 & 68566 & 274262 \\
\hline
\end{tabular}

It was found out that the optimization by PSO or GA allows the enterprise to save active power extra 16234 or 17943 euros, correspondingly, each year. In terms of this value, the divergence between these algorithms is $10 \%$. Table 4 represents the payback of the deep compensation. The analysis shows that the deep compensation pays off in about 1 year and 7 months. Table 4 demonstrates that the advantage of the choice of the PSO algorithm instead GA is increasing every year.

The results obtained by solving the optimization problem with minimization the cable cross-section for the whole system (Equation 2\&3) are shown in Table 5. Table 4 shows that GA and PSO found the solutions similar to efficiency and PSO outperformed GA. In the case of using the PSO solution, the total cost saving is 113 thousand euros in comparison with the variant that is not optimized. It is 27 thousand better than the GA solution. 
Table 4. The Payback of the Deep Compensation

\begin{tabular}{cccccc}
\hline Time (years) & $\begin{array}{c}\text { Criterion } Z_{1} \text { without } \\
\text { optimization }(€)\end{array}$ & $\begin{array}{c}\text { GA criterion } Z_{1} \\
(€)\end{array}$ & GA profit $(€)$ & $\begin{array}{c}\text { PSO criterion } Z_{1} \\
(€)\end{array}$ & PSO profit (€) \\
\hline 1 & 10814 & 12238 & -1424 & 11968 & -1154 \\
1 and 7 months & 17122 & 17362 & -240 & 16967 & 155 \\
2 & 21627 & 21022 & 605 & 20538 & 1089 \\
3 & 32440 & 29807 & 2633 & 29109 & 3331 \\
4 & 43254 & 38591 & 4663 & 37689 & 5574 \\
5 & 54068 & 47375 & 6693 & 46251 & 7818 \\
6 & 64881 & 56160 & 8721 & 54821 & 10060 \\
7 & 75694 & 64945 & 10749 & 63391 & 12303 \\
8 & 86507 & 73730 & 12777 & 71961 & 14546 \\
10 & 97320 & 82515 & 14805 & 80531 & 16789 \\
\hline
\end{tabular}

Table 5. The Results from Equation 2 and 3 with Minimization the Cable Cross-Section

\begin{tabular}{cccccc}
\hline Algorithm & Criterion $Z_{2}(€)$ & $\begin{array}{c}\text { Active power losses } \\
(\mathrm{kW})\end{array}$ & $\begin{array}{c}\text { Total power of } \\
\text { RPCUs (kvar) }\end{array}$ & $\begin{array}{c}\text { Cable lines } \\
\cos (€)\end{array}$ & $\begin{array}{c}\operatorname{tg} \varphi \\
\text { without optimization }\end{array}$ \\
GA & 373654 & 324.4 & 0 & 330400 & 0.55 \\
PSO & 287537 & 269.6 & 8976 & 248000 & 0.14 \\
\hline
\end{tabular}

\section{CONCLUSION}

The optimal placement of reactive power sources was considered from two points of view. In the first case, the power lines are laid down since it is possible to minimize the active power losses only. In the second case, it is possible to change the cross-sections of the supply lines to minimize the active power losses and the volume of the cable lines. The financial costs of the active power losses by 4 years, the RPCUs installed and the cable volume was used as the single optimization criterion. In the first case, the criterion can be improved by $12.9 \%$ ( 44.5 thousand euros for the whole system of the eight sections). In the second case, the criterion can be improved by $30.2 \%$ (113 thousand euros for the whole system of the eight sections).

The population-based stochastic algorithms can be applied to optimization problems in the field of power supply systems and smart grid easily and quickly due to their flexibility and ability to explore the decision space automatically. Despite the fact that the GA and the PSO algorithms are very different in terms of the logic of the internal operations, they were applied to the same model of the optimization problem without any changes in the model. Thus, a power engineer can develop the mathematical model, during optimization algorithms can be configured and applied by an artificial intelligence specialist. This approach makes it easy to combine the competence of different specialists in a system to increase the productivity of their research and development.

In this study, PSO showed the better results than GA for the both variants of the optimization problem considered. The difference between the results of PSO and GA is not too high. As population algorithm is easy to apply, it is reasonable to use various population-based algorithms for increasing the probability of obtaining the global optimum.

\section{REFERENCES}

[1] A. H. Mantawy, et al., "Integrating Genetic Algorithms, Tabu Search, and Simulated Annealing for the Unit Commitment Problem," IEEE Transactions on Power Systems, vol/issue: 14(3), pp. 829-836, 1999. DOI: 10.1109/59.780892.

[2] P. Paterni, et al., "Optimal Location of Phase Shifters in the French Network by Genetic Algorithm," IEEE Transactions on Power Systems, vol/issue: 14(1), pp. 37-42, 1999. DOI: 10.1109/59.744481.

[3] D. Dervani and J. P. Roselyn, "Genetic algorithm based reactive power dispatch for voltage stability improvement," International Journal of Electrical Power \& Energy Systems, vol/issue: 32(10), pp. 1151-1156, 2010. DOI: 10.1016/j.ijepes.2010.06.014.

[4] M. I. Azim and M. F. Rahman, "Genetic Algorithm Based Reactive Power Management by SVC," International Journal of Electrical and Computer Engineering, vol/issue: 4(2), pp. 200-206, 2014.

[5] V. Manusov, et al., "Population-based Algorithms for Optimization of the Reactive Power Distribution and Selection of the Cable Cross-section in the Power-Supply Systems," Applied Mechanics and Materials, vol. 792, pp. 230-236, 2015. DOI: 10.4028/www.scientific.net/AMM.792.230.

[6] M. De and S. K. Goswami, "Optimal Reactive Power Procurement with Voltage Stability Consideration in Deregulated Power System,” IEEE Transaction on Power Systems, vol/issue: 29(5), pp. 2078-2086, 2014. DOI: 10.1109/TPWRS.2014.2308304. 
[7] J. J. Jamian, et al., "A New Particle Swarm Optimization Technique in Optimizing Size of Distributed Generation," International Journal of Electrical and Computer Engineering, vol/issue: 1(1), pp. 137-146, 2012.

[8] P. V. Matrenin and V. G. Sekaev, "Sistemnoe opisanie algoritmov roevogo intellekta [Systems approach to swarm intelligence]," Programmnaja inzhenerija, vol. 12, pp. 39-45, 2013. [in Russian].

[9] M. Melanie, "An Introduction to Genetic Algorithms," Cambridge, MA, MIT Press, 1996.

[10] J. H. Holland, "Adaptation in natural and artificial systems," University of Michigan Press, Ann Arbor, 1975.

[11] Y. Zhu Y and X. Tang, "Overview of swarm intelligence," Computer Application and System Modeling (ICCASM), vol. 9, pp. 400-409, 2010.

[12] J. Kennedy and R. C. Eberhart, "Particle swarm optimization," in Proc. IEEE International Conference on Neural Networks, Piscataway, NJ, pp. 1942-1948, 1995. DOI: 10.1109/ICNN.1995.488968.

[13] M. Pedersen and A. Chipper, "Simplifying Particle Swarm Optimization," Applied Soft Computing, vol/issue: 10(2), pp. 618-628, 2010.

[14] P. V. Matrenin and V. G. Sekaev, "Particle Swarm optimization with velocity restriction and evolutionary parameters selection for scheduling problem," in Proc. 2015 International Siberian Conference Control and Communications (SIBCON), Omsk, pp. 1-5, 2015. DOI: 10.1109/SIBCON.2015.7147143.

[15] R. C. Eberhart and Y. Shi, "Particle swarm optimization: developments, applications and resources," in Proc. Congress on Evolutionary Computation, vol. 1, pp. 81-86, 2001. DOI: 10.1109/CEC.2001.934374. 